\title{
Relationship between quality of life and unprotected anal intercourse among Chinese men who have sex with men: a cross-sectional study
}

Yaxin Zhu, Jie Liu, Bo Qu*, Bingxue Hu and Yang Zhang

\begin{abstract}
Background: The prevalence of unprotected anal intercourse (UAI) is high among Chinese men who have sex with men (MSM). As important aspects of quality of life (QOL), psychological health and social relationships have been found to be associated with UAI among MSM, which was of great concern for intervening on UAI.

Methods: We conducted a cross-sectional study in Zhengzhou, Henan province, and in Huludao, Liaoning province, China, to measure quality of life (QOL) using the brief version of the World Health Organization Quality of Life (WHOQOL-BREF). Cronbach's a coefficient was used to test the internal consistency of the questionnaire items, and construction validity was assessed by exploratory factor analysis. T-test, chi-square test and multivariate logistic analysis were carried out to test the relationship between quality of life and unprotected anal intercourse.

Results: The questionnaires of 320 of the 373 men who have sex with men that were surveyed met our validity criteria (response rate: $85.8 \%$ ). A total of 161 subjects (50.3\%) reported having unprotected anal sex in the last 6 months. The results of univariate analysis indicated that having unprotected anal sex was significantly associated with psychological health (PSYCH), social relationships (SOCIL), age, and marital status ( $P$-value $<0.05$ ). The 3 items ("Negative feelings", "Hopeness on your life", and "Be able to concentrate") in the PSYCH subscale were associated with UAl significantly ( $P$-value $<0.05$ ). Multivariate analysis showed that psychological health (adjusted odd ratio (AOR) $0.979,95 \%$ confidence interval (CI) 0.961-0.998) was independent factor affecting the likelihood of having UAl in the population of Chinese MSM, and participants who aged 45 or more had higher odds of UAI (AOR 3.986, $95 \% \mathrm{Cl}$ 1.199-13.255).

Conclusions: WHOQOL-BREF was acceptable for evaluating the quality of life of MSM. Psychological health, as one important aspect of quality of life, was negatively associated with unprotected anal intercourse. The finding underscored the needs to incorporate the strategies on improving psychological health into the UAI intervention to reduce the HIV transmission among MSM.
\end{abstract}

Keywords: Men who have sex with men, Unprotected anal intercourse, Quality of life, WHOQOL-BREF, HIV/AIDS

\section{Background}

HIV epidemics have been declining in many countries and in various populations, yet the incidence is increasing within men who have sex with men (MSM) population in most countries, including China [1-3]. Although the national HIV sentinel surveillance data showed that

\footnotetext{
*Correspondence: qubo6666@163.com

Department of Health Statistics, School of Public Health, China Medical University, No. 77 Puhe Road, Shenyang North New Area, Shenyang,

Liaoning Province 110122, People's Republic of China
}

the HIV-positive rate of risk groups, including injection drug users, female sex workers, and pregnant women, have dropped somewhat or maintained a low level, the rate among Chinese MSM has increased by more than 8 fold from $0.9 \%$ in 2003 to $7.3 \%$ in 2013 [4]. Furthermore, of newly diagnosed cases of HIV/AIDS each year, the percentage of male homosexually transmitted cases has increased from $2.5 \%$ in 2006 to $21.4 \%$ in 2013 [4]. Thus, MSM remains an important driver of Chinese HIV/AIDS epidemic. 
Globally, unprotected anal intercourse (UAI) has been indicated as a major risk behavior for HIV infection among MSM [5-9]. Meta-analysis of 82 studies showed that the UAI rate was $53 \%$ among Chinese MSM, and the high rate may result in a serious HIV epidemic [10]. Previous studies have suggested that intervention efforts aimed at reducing UAI may facilitate a reduction in HIV transmission [11, 12]. Such factors, including mental issue [13], substance or alcohol use [14], HIV knowledge [15], social stigma and support [16], have been proven determinants of UAI. Wim et al. reported that having a history of mental health problems, such as depressive symptoms, was associated with higher rates of UAI and HIV transmission among MSM [13, 17]. A study on MSM showed that less-supportive social relationship was related to higher rates of unsafe sex [16]. And taking various influencing factors on HIV-risk behavior into account for proposing intervention strategies could produce more sustained reduction in HIV transmission among MSM [7, 18, 19]. Thus, surveying mental health and social roles, which are important aspects of quality of life (QOL) [20], should be of great concern for intervention aimed at reducing the risk of HIV [9].

Quality of life (QOL) is regarded as a good measure of overall health [21]. Previous studies on QOL have been conducted among MSM population, and indicated that surveying the QOL of the MSM can inform us of their physical, psychological, cognitive and social functions according to the individuals' perceptions their health [22-24]. Dey et al. also suggest that the association between QOL and health-risk behavior, including UAI, cigarette smoking, cannabis use, may provide insight into the reasons why a person may engage in these particular behaviors [25]. And Bouhnik et al. observed that poor mental health-related QOL was associated with UAI in French HIV-infected gay men [23].

In the present study, the brief version of World Health Organization quality of life (WHOQOL-BREF) was used to assess the QOL. WHOQOL-BREF is an international cross-culturally comparable quality of life assessment instrument to assess the whole health conditions [26], and this scale not only provides a direct quantitative indication of the physical and mental health states, but also provides information about social relationships and environment [27]. There have been several studies on evaluating QOL of MSM using WHOQOL-BREF [20, 28]. The Chinese version of WHOQOL-BREF has proven reliable and valid among MSM population [29, 30].

The aim of the present study was to explore the relationship between QOL and UAI within the Chinese MSM population, and provide the local Center for Disease Control and Prevention (CDC) with some suggestion on UAI intervention to reduce the HIV transmission among MSM.

\section{Methods}

\section{Ethics statement}

This study was approved by the Bioethics Advisory Commission of China Medical University. Written informed consent was obtained from each of the participants.

\section{Participant recruitment}

We conducted a cross-sectional study from April to June of 2014 in Zhengzhou, Henan province and in Huludao, Liaoning province, China. Respondents were recruited from gay bars, gay saunas, and parks. Participants were eligible if they were at least 18 years old and had anal sex with men in the last 6 months before our study. There were 320 valid questionnaires obtained from the 373 MSM surveyed (response rate: $85.8 \%$ ).

\section{Procedures}

Eligible MSM completed the questionnaire in a private room using the standardized version on their own. And if they had difficulty in understanding some questions as they filled out the questionnaire, the trained interviewers would provide an explanation. All subjects were given the option to decline participation at any time, and an incentive of 50 Yuan (about 9 USD) was given for participating in the survey.

\section{Survey questionnaire}

Our questionnaire consisted of two parts: a sociodemographic portion and the WHOQOL-BREF. The socio-demographic characteristics included age, marital status, education level, vocation and monthly income. The WHOQOL-BREF includes 24 items in four domains, namely physical health (PHYS), psychological health (PSYCH), social relationships (SOCIL), and environment (ENVIR) and 2 items about overall QOL and general health. The 26 items are scored from 1 to 5 , and the scores of four domains are transformed into 0 to 100 , with higher scores indicating better QOL. Unprotected anal intercourse (UAI) was defined as having had at least one incident of anal intercourse without a condom with any male partner during the previous 6 months.

\section{Statistical methods}

Cronbach's $\alpha$ coefficient was used to test the internal consistency of the WHOQOL-BREF items, and construction validity was assessed by exploratory factor analysis. $T$-test, $X^{2}$ test and multivariate regression analysis were carried out to test the relationship between QOL and UAI rate. All significant variables in the univariate analysis were then entered into a multivariate regression model, and stepwise method was used to select risk factors of UAI in the model. The data were analyzed using SPSS, version 17.0 (SPSS Inc., Chicago, IL, USA) for 
Windows. A $P$-value $<0.05$ was considered statistically significant.

\section{Results}

\section{Socio-demographic characteristics}

The median age of this population was 26 years (range 18-63 years). 61 participants $(19.0 \%)$ were married. The socioeconomic characteristics of the subjects are shown in Table 1.

\section{Reliability and validity analysis}

The overall Cronbach's $\alpha$ coefficient for our analysis was 0.926. The results of Kaiser-Meyer-Olkin (0.917) and the Bartlett's test of sphericity $\left(\chi^{2}=4033.378, P\right.$ value $<0.001)$ indicated that the samples in this study are suitable for factor analysis. Exploratory factor analysis showed that the accumulative variance contribution rate was $59.88 \%$.

\section{Univariate analysis of factors associated with UAI}

A total of 161 subjects (50.3\%) reported having UAI. The univariate analysis (Table 1 ) indicated that engaging in UAI was statistically associated with older age and being married respectively ( $P$-value $<0.05)$.

The PSYCH and SOCIL subscale were found to be inversely associated with practicing UAI $(P$-value $<0.05)$ (Table 2). There are 3 items in the PSYCH subscale, which were "Hopeness on your life", "Negative feelings", and "Be able to concentrate", respectively, were associated with UAI significantly $(P$-value $<0.05)$. The item "Satisfy with your sex life", which is in the domain of SOCIL, was also significant factor of having UAI ( $P$ value $<0.05)$. The results are shown in Table 3.

\section{Multivariate analysis of factors associated with UAI}

PSYCH score was significant factor affecting UAI in the multivariate logistic regression model (AOR 0.979, $95 \%$ CI 0.961-0.998). Compared with those participants who aged less than 45 , the participants aged 45 or more had significant higher odds of engaging in UAI (AOR 3.986, 95 \% CI 1.199-13.255). The results are shown in Table 4.

\section{Discussion}

$50.3 \%$ of subjects reported having UAI. This result is comparable to the reported rates of UAI in Chinese

Table 1 Demographic characteristics of MSM population ( $N=320)$

\begin{tabular}{|c|c|c|c|c|c|}
\hline \multirow[t]{2}{*}{ Variable } & \multirow[t]{2}{*}{ Total } & \multicolumn{2}{|l|}{ UAl } & \multirow[t]{2}{*}{$x^{2}$} & \multirow[t]{2}{*}{$P$-value } \\
\hline & & Yes & No & & \\
\hline Age & & & & 10.104 & 0.018 \\
\hline$<=25$ & $124(38.8 \%)$ & $57(46.0 \%)$ & $67(54.0 \%)$ & & \\
\hline $26-35$ & $131(40.9 \%)$ & $63(48.1 \%)$ & $68(51.9 \%)$ & & \\
\hline $36-44$ & 43 (13.4\%) & 23 (53.5 \%) & 20 (46.5 \%) & & \\
\hline$>=45$ & $22(6.9 \%)$ & $18(81.8 \%)$ & $4(18.2 \%)$ & & \\
\hline Marital status & & & & 4.329 & 0.037 \\
\hline Single & $259(80.9 \%)$ & $123(47.5 \%)$ & $136(52.5 \%)$ & & \\
\hline Married & $61(19.1 \%)$ & $38(62.3 \%)$ & $23(37.7 \%)$ & & \\
\hline Education & & & & 3.062 & 0.216 \\
\hline Middle school or lower & $54(16.9 \%)$ & $33(61.1 \%)$ & $21(38.9 \%)$ & & \\
\hline Secondary technical school or high school & $90(28.1 \%)$ & $44(48.9 \%)$ & $46(51.1 \%)$ & & \\
\hline College or higher & $176(55.0 \%)$ & $84(47.7 \%)$ & $92(52.3 \%)$ & & \\
\hline Vocation & & & & 3.661 & 0.300 \\
\hline White collar & $66(20.6 \%)$ & $31(47.0 \%)$ & 35 (53.0 \%) & & \\
\hline Blue collar & $217(67.8 \%)$ & $106(48.8 \%)$ & $111(51.2 \%)$ & & \\
\hline unemployed & $19(5.9 \%)$ & $12(63.2 \%)$ & $7(36.8 \%)$ & & \\
\hline Student & $18(5.6 \%)$ & $12(66.7)$ & $6(33.3)$ & & \\
\hline Monthly income & & & & 4.805 & 0.091 \\
\hline$<=2000$ Yuan & $87(27.2 \%)$ & $43(49.4 \%)$ & $44(50.6 \%)$ & & \\
\hline 2001-2999 Yuan & $146(45.6 \%)$ & $82(56.2 \%)$ & $64(43.8 \%)$ & & \\
\hline$>=3000$ Yuan & 87 (27.2 \%) & $36(41.4 \%)$ & $51(58.6 \%)$ & & \\
\hline
\end{tabular}


Table 2 The relationship between Quality of life (QOL) and UAI among MSM population by univariate analysis

\begin{tabular}{lllll}
\hline Domain & \multicolumn{2}{l}{ UAl } & \multirow{2}{*}{ t } & P-value \\
\cline { 2 - 3 } & Yes & No & & \\
\cline { 1 - 3 } PHYS (physical health) & $67.32 \pm 14.69$ & $70.48 \pm 13.91$ & 1.942 & 0.058 \\
PSYCH (psychological health) & $59.76 \pm 17.09$ & $64.94 \pm 14.88$ & 2.891 & 0.004 \\
SOCIL (social relationships) & $63.87 \pm 16.67$ & $67.40 \pm 14.84$ & 2.000 & 0.046 \\
ENVIR (environment) & $55.57 \pm 16.60$ & $57.70 \pm 14.00$ & 1.242 & 0.215 \\
Q1 (general QOL) & $3.71 \pm 0.87$ & $3.74 \pm 0.82$ & 0.295 & 0.768 \\
Q2 (general health) & $3.46 \pm 0.88$ & $3.64 \pm 0.75$ & 1.853 & 0.065 \\
\hline
\end{tabular}

MSM = men who have sex with men

$\mathrm{UAI}=$ unprotected anal intercourse

MSM (53 \%, 95 \% CI: 51 to $56 \%$ ) and in MSM in 21 US cities (54 \%) [10, 31]. Our study found that psychological health and social relationships as two out of four domains of QOL were statistically significant factors of UAI among MSM in our study. The reliability and validity of the WHOQOL-BREF were acceptable. Univariate analysis showed that lower PSYCH and SOCIL subscale scores were significantly related to UAI $(P$-value $<0.05)$. And the older MSM and being married were associated with UAI respectively $(P$-value $<0.05)$. Multivariate analysis showed that PSYCH (AOR $=0.979 ; 95 \%$ CI: 0.961 to 0.998 ) and age 45 or more (AOR 3.986, $95 \% \mathrm{CI}$ 1.199-13.255) were independent factors influencing UAI in Chinese MSM $(P$-value $<0.05)$.

In line with other studies, we found that poor psychological health was significantly associated with engaging in UAI (P-value <0.05) $[9,23]$. More specifically, the lower scores of the items "Negative feelings", "Hopeness on your life", and "Be able to concentrate" were determinants of UAI, respectively ( $P$-value $<0.05)$. Due to experiencing discrimination about sexual orientation by family and other institutions, many Chinese MSM had encountered stigma, which led to mood, anxiety disorders and depression more common in MSM than in men in the general population [32, 33]. Moreover,

Table 3 The influence of psychological health and social relationships on UAI among MSM population by univariate analysis

\begin{tabular}{lllll}
\hline & \multicolumn{1}{l}{ UAl } & t & P-value \\
\cline { 2 - 3 } Domain $^{\text {a }}$ & No & & \\
\hline PSYCH (psychological health) & & & & \\
Hopeness on your life & $3.41 \pm 1.00$ & $3.69 \pm 0.91$ & 2.636 & 0.009 \\
Negative feelings & $3.03 \pm 0.89$ & $3.45 \pm 0.97$ & 4.067 & 0.000 \\
Be able to concentrate & $3.45 \pm 0.94$ & $3.71 \pm 0.92$ & 2.539 & 0.012 \\
SOCIL (social relationships) & & & & \\
Satisfy with your sex life & $3.40 \pm 0.82$ & $3.60 \pm 0.81$ & 2.267 & 0.024 \\
\hline
\end{tabular}

MSM = men who have sex with men

$\mathrm{UAI}=$ unprotected anal intercourse

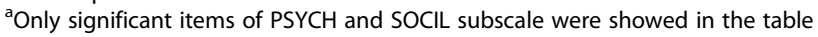

Table 4 Multivariate logistic regression models showing risk factors of $\mathrm{UAl}^{\mathrm{a}}$

\begin{tabular}{lll}
\hline Factor & COR $(95 \% \mathrm{Cl})^{\mathrm{b}}$ & AOR $(95 \% \mathrm{Cl})^{\mathrm{c}}$ \\
\hline $\begin{array}{l}\text { Sociodemographics } \\
\text { Age }\end{array}$ & & \\
$\quad<=25$ & 1 & 1 \\
$26-35$ & $1.089(0.666-1.781)$ & $0.998(0.601-1.658)$ \\
$36-44$ & $1.352(0.674-2.710)$ & $1.050(0.486-2.267)$ \\
$>=45$ & $5.289(1.692-16.531)^{*}$ & $3.986(1.199-13.255)^{*}$ \\
Marital status & & \\
$\quad$ Single & 1 & 1 \\
$\quad$ Married & $1.827(1.031-3.238)^{*}$ & $1.492(0.774-2.877)$ \\
QOL & & \\
PSYCH (psychological & $0.980(0.966-0.994)^{*}$ & $0.979(0.961-0.998)^{*}$ \\
health) & & \\
SOCIL (social relationships) & $0.986(0.972-1.000)^{*}$ & $1.003(0.984-1.022)$ \\
\hline
\end{tabular}

${ }^{a}$ Only factors significant in univariate analysis were included in the multivariate logistic regression model

bUnivariate logistic regression model

${ }^{c}$ Multivariate logistic regression model

${ }^{*} p<0.05$

$C O R$ crude odds ratio, $A O R$ adjusted odds ratio, $\mathrm{Cl}$ confidence interval

increasing evidence has shown that compared to heterosexuals, gays exhibited elevations in hopelessness caused by the stressors that sexual minorities confront [34-36]. Safren et al. also indicated that mental health problems may compromise the impact of HIV prevention programs [37]. Behavioral interventions combined with mental health treatment have been shown to increase the effectiveness of reducing UAI and HIV prevention programs among MSM and HIV-infected individuals [37-39]. And previous studies implied that communitybased or structural interventions that reduce sexual minority prejudices, in combination with individual-based interventions that address negative beliefs about life, may help to alleviate the high rates of mental health problems among MSM [34, 37]. Our results suggest that improving psychological health is an aspect that should be incorporated into UAI reduction-intervention strategies to facilitate HIV prevention among Chinese MSM.

Our study also showed that weaker social relationships were associated with a higher likelihood of practicing UAI in the MSM population ( $P$-value $<0.05)$. Given homosexuality is generally viewed as unacceptable in Chinese culture, MSM tend to report more social isolation, and avoid entering close relationships for fear of others' discovering their sex orientation, which led this population to limited access to the public health care system aimed at gay community [34, 40, 41]. Previous study also suggested that sexual risk prevention interventions should take addressing their capacity to build adequate and supportive social relationships into account to enhance HIV prevention programs among 
MSM population [16]. Furthermore, we also found that lower score of the "Satisfy with your sex life" item which included in the SOCIL domain was significantly related to engaging UAI. Tsui et al. also found that lack of sexual pleasure was significantly associated with UAI among MSM in Shenzhen and HongKong [42]. A study on HIV-positive MSM showed that sex toy users reported higher levels of sexual satisfaction, and suggested that the effect of sex toys as tools on enhancing sexual pleasure and reducing HIV transmission should be explored in further studies [43].

Our results also found that older Chinese MSM were more likely to engage in UAI than younger MSM, which is consistent with several other studies of Chinese MSM $[44,45]$. In addition, according to the national report on HIV/AIDS cases, new HIV cases among the higher age group increased noticeably $[4,46]$. There could be several reasons to explain why the UAI rate of Chinese older MSM was higher. Firstly, few HIV intervention efforts aimed at reducing UAI have focused on older MSM, for the inaccurate belief that older men are not sexually active [45]. Secondly, ageism and homosexual stigma could cause older MSM to hide their homosexual activity, thus increasing the difficulty of reaching them by prevention intervention $[47,48]$. Thus, targeted interventions on reducing UAI in this HIV-risk subgroup could be of great need.

In sum, UAI intervention programs targeting MSM need to be evidence-based and would benefit from sound research support. Our study also had some limitations. Firstly, random sampling was not performed, and the sampling was venue based in this study. Secondly, the study was conducted in only two Chinese cities, and the results may not be representative of all, or even most, Chinese MSM. And in our study, the questionnaire in which more than $20 \%$ of items were left unanswered was considered to be invalid. $15 \%$ of the respondents only completed below $80 \%$ of the questionnaires, probably because they may be not willing to expose their personal information, such as age, sexual behavior. This may be why nearly $15 \%$ of surveys did not meet the criteria of "valid". Further studies in more areas of China will determine whether these findings can be generalized.

\section{Conclusions}

Poorer psychological health and social relationships, as the important aspects of QOL, were found in the present study to be associated with higher likelihood of engaging in UAI, and psychological health played a larger role. It therefore appears necessary to incorporate improved psychological health into future intervention strategies to prevent UAI in MSM.

\section{Ethical approval and consent to participate}

This study was approved by the Bioethics Advisory Commission of China Medical University. Written informed consent was obtained from each of the participants.

\section{Consent to publish}

Not applicable.

\section{Availability of data and materials}

All data is available in the paper. Data will be shared upon request and it is subjected to the data protection regulations.

\section{Abbreviations}

UAI: unprotected anal intercourse; QOL: quality of life; MSM: men who have sex with men; PSYCH: psychological health; PHYS: physical health;

PSYCH: psychological health; SOCIL: social relationships; ENVIR: environment.

\section{Competing interests}

The authors declare that they have no competing interests.

\section{Authors' contributions}

$\mathrm{BQ}$ designed the research. $Y X Z, B X H$, and $Y Z$ participated in the data collection. YXZ wrote the manuscript. JL and YZ participated in data analysis. All authors read and approved the final manuscript.

\section{Acknowledgements}

We are particularly grateful to all the participants in the survey and kindly responded to the questionnaire. The authors would like to thank Shijie Liang and Jing Guo who helped in the data collection.

\section{Funding}

This study was supported by the National Natural Science Foundation of China (Grant Number 81273186 and 71473269). The National Natural Science Foundation of China had no role in the design of the study, in the collection, analysis or interpretation of data, or in the writing of the manuscript.

Received: 25 December 2015 Accepted: 4 May 2016

Published online: 10 May 2016

\section{References}

1. Wirtz AL, Kirey A, Peryskina A, Houdart F, Beyrer C. Uncovering the epidemic of HIV among men who have sex with men in Central Asia. Drug Alcohol Depend. 2013;132 Suppl 1:S17-24.

2. Zhang L, Zhang D, Yu B, Wang S, Liu Y, Wang J, et al. Prevalence of HIV Infection and Associated Risk Factors among Men Who Have Sex with Men (MSM) in Harbin, P.R. China. PLoS One. 2013;8:e58440.

3. Beyrer C, Sullivan P, Sanchez J, Baral SD, Collins C, Wirtz AL, et al. The increase in global HIV epidemics in MSM. AIDS. 2013;27:2665-78.

4. State Council AIDS Working Committee Office (SCAWCO). 2014 China AIDS Response Progress Report. Beijing, China: National Health and Family Planning Commission of The People's Republic of China. June, 2014: 1-30. http://www.unaids.org.cn/en/index/Document_view.asp?id=859. Accessed on 27 January, 2015. [Context Link]

5. Li J, Lau JT, Gu J, Hao C, Lai CH. Event-Specific Risk Factors Predicting Episodes of Unprotected Anal Intercourse with Male Nonregular Partners among Men Who Have Sex with Men Using Case-Crossover Study Design. Biomed Res Int. 2014;2014:475195.

6. Wang K, Yan H, Liu Y, Leng Z, Wang B, Zhao J. Increasing prevalence of HIV and syphilis but decreasing rate of self-reported unprotected anal intercourse among men who had sex with men in Harbin, China: results of five consecutive surveys from 2006 to 2010. Int J Epidemiol. 2012;41:423-32.

7. McDaid LM, Hart GJ. Sexual risk behavior for transmission of HIV in men who have sex with men: recent findings and potential interventions. Curr Opin HIV AIDS. 2010;5:311-5. 
8. Hart GJ, Elford J. Sexual risk behaviour of men who have sex with men: emerging patterns and new challenges. Curr Opin Infect Dis. 2010;23:39-44.

9. Mayer KH, Bekker LG, Stall R, Grulich AE, Colfax G, Lama JR. Comprehensive clinical care for men who have sex with men: an integrated approach. Lancet. 2012;380:378-87.

10. Wu J, Hu Y, Jia Y, Su Y, Cui H, Liu H, et al. Prevalence of Unprotected Anal Intercourse among Men Who Have Sex with Men in China: An Updated Meta-Analysis. PLoS One. 2014;9:e98366.

11. Prestage G, Mao L, Fogarty A, Van de Ven P, Kippax S, Crawford J, et al. How has the sexual behavior of gay men changed since the onset of AIDS: 1986-2003. Aust N Z J Public Health. 2005;29:530-5.

12. Jin F, Crawford J, Prestage GP, Zablotska I, Imrie J, Kippax SC, et al. HIV risk reduction behaviors in gay men: Unprotected anal intercourse, risk reduction behaviors, and subsequent HIV infection in a cohort of homosexual men. AIDS. 2009:23:243-52.

13. Wim VB, Christiana N, Marie L. Syndemic and other risk factors for unprotected anal intercourse among an online sample of Belgian HIV negative men who have sex with men. AIDS Behav. 2014;18:50-8.

14. Dolezal C, Carballo-Diéguez A, Nieves-Rosa L, Díaz FC. Substance use and sexual risk behavior: understanding their association among four ethnic groups of Latino men who have sex with men. J Subst Abuse. 2000;11:323-36.

15. Ruan S, Yang H, Zhu Y, Ma Y, Li J, Zhao J, et al. HIV prevalence and correlates of unprotected anal intercourse among men who have sex with men, Jinan, China. AIDS Behav. 2008;12:469-75.

16. Buttram ME, Kurtz SP, Surratt HL. Substance use and sexual risk mediated by social support among Black men. J Community Health. 2013;38:62-9.

17. Mimiaga MJ, Noonan E, Donnell D, Safren SA, Koenen KC, Gortmaker S, et al. Childhood Sexual Abuse Is Highly Associated With HIV Risk-Taking Behavior and Infection Among MSM in the EXPLORE Study. J Acquir Immune Defic Syndr. 2009;51:340-8.

18. Lau JT, Feng TJ, Liu XL, Gu J, Tsui HY, Hong FC, et al. Associations between Cognitive, Sociocontextual, and Affective Variables and Unprotected Anal Intercourse among Men Who Have Sex with Men-A Comparative Study Conducted in Two Chinese Cities. Biomed Res Int. 2014;2014:970975.

19. Coates TJ, Richter L, Caceres C. Behavioural strategies to reduce HIV transmission: how to make them work better. Lancet. 2008;372:669-84.

20. Chen JP, Han MM, Liao ZJ, Dai ZZ, Liu L, Chen H, et al. HIV-related behaviors, social support and health-related quality of life among men who have sex with men and women (MSMW): a cross-sectional study in Chongqing, China. PLoS One. 2015;10:e0118651.

21. Croog SH, Levine S. Quality of life and health care interventions. In: Freeman HE, Levine S, editors. Handbook of Medical Sociology. 4th ed. New Jersey: Prentice Hall; 1989. p. 508-28.

22. Liu J, Qu B, Hu B, Jiang N, Wang D. The Quality of Life of Men Who Have Sex with Men in China: Reliability and Validity Testing of the SF-36 Questionnaire. PLoS One. 2013;8:e83362.

23. Bouhnik $A D$, Préau $M$, Schiltz MA, Peretti-Watel $P$, Obadia $Y$, Lert $F$, et al Unsafe sex with casual partners and quality of life among HIV-infected gay men: evidence from a large representative sample of outpatients attending French hospitals (ANRS-EN12-VESPA). J Acquir Immune Defic Syndr. 2006;42: 597-603.

24. Kelly-Campbell RJ, Atcherson SR. Perception of quality of life for adults with hearing impairment in the LGBT community. J Commun Disord. 2012:45: 367-77.

25. Dey M, Gmel G, Studer J, Mohler-Kuo M. Health-risk behaviors and quality of life among young men. Qual Life Res. 2014;23:1009-17.

26. The WHOQOL Group. Development of the World Health Organization WHOQOL-BREF quality of life assessment. Psychol Med. 1998;28:551-8.

27. Chen JP, Wang H, Liu L. Quality of life and related social support for men who have sex with men among university students in Chongqing, China. Zhonghua Liu Xing Bing Xue Za Zhi. 2013;34:888-92 [Article in Chinese].

28. Ghorayeb DB, Dalgalarrondo P. Homosexuality: mental health and quality of life in a Brazilian socio-cultural context. Int J Soc Psychiatry. 2011;57:496-500.

29. Zhang Y, Qu B, Lun S, Wang D, Guo Y, Liu J. Quality of life of medical students in China: a study using the WHOQOL-BREF. PLoS One. 2012;7: e49714

30. Xia P, Li N, Hau KT, Liu C, Lu Y. Quality of life of Chinese urban community residents: a psychometric study of the mainland Chinese version of the WHOQOL-BREF. BMC Med Res Methodol. 2012;27:37.

31. Finlayson TJ, Le B, Smith A, Bowles K, Cribbin M, Miles I, et al. HIV risk, prevention, and testing behaviors among men who have sex with
men-National HIV Behavioral Surveillance System, 21 US cities, United States, 2008. MMWR. 2011;60:1-34.

32. Feng $Y$, Wu Z, Detels R, Qin G, Liu L, Wang $X$, et al. HIV/STD prevalence among men who have sex with men in Chengdu, China and associated risk factors for HIV infection. J Acquir Immune Defic Syndr. 2010;53 Suppl 1:574-80.

33. Liu JX, Choi K. Experiences of social discrimination among men who have sex with men in Shanghai, China. AIDS Behav. 2006;10 Suppl 4:S25-33.

34. Hatzenbuehler ML. How Does Sexual Minority Stigma "Get Under the Skin"? A Psychological Mediation Framework. Psychol Bull. 2009;135:707-30.

35. van Heeringen C, Vincke J. Suicidal acts and ideation in homosexual and bisexual young people: A study of prevalence and risk factors. Soc Psychiatry Psychiatr Epidemiol. 2000;35:494-9.

36. Walls NE, Freedenthal S, Wisneski H. Suicidal ideation and attempts among sexual minority youths receiving social services. Soc Work. 2008:53:21-9.

37. Safren SA, Blashill AJ, O'Cleirigh CM. Promoting the sexual health of MSM in the context of comorbid mental health problems. AIDS Behav. 2011;15 Suppl 1:S30-4.

38. Crepaz N, Lyles CM, Wolitski RJ, Passin WF, Rama SM, Herbst JH, et al. Do prevention interventions reduce HIV risk behaviors among people living with HIV? A meta-analytic review of controlled trials. AIDS. 2006;20:143-57.

39. Sikkema KJ, Watt MH, Drabkin AS, Meade CS, Hansen NB, Pence BW. Mental health treatment to reduce HIV transmission risk behavior: a positive prevention model. AIDS Behav. 2010;14:252-62.

40. Liu J, Ou B, Ezeakile MC, Zhang Y. Factors Associated with Unprotected Anal Intercourse among Men Who Have Sex with Men in Liaoning Province, China. PLoS One. 2012;7:e50493.

41. Liu H, Yang H, Li X, Wang N, Liu H, Wang B, et al. Men who have sex with men and human immunodeficiency virus/sexually transmitted disease control in China. Sex Transm Dis. 2006;33:68-76.

42. Tsui HY, Lau JT, Feng T, Hong F, Cai Y, Zhou H, et al. Sexual Dysfunction and Unprotected Anal Intercourse Among Men Who Have Sex With Men in Two Chinese Cities. J Sex Marital Ther. 2014;40:139-48.

43. Satinsky S, Rosenberger JG, Schick V, Novak DS, Reece M. USA study of sex toy use by HIV-positive men who have sex with other men: implications for sexual health. Int J STD AIDS. 2011:22:442-8.

44. Tang W, Huan X, Mahapatra T, Tang S, Li J, Yan H, et al. Factors Associated with Unprotected Anal Intercourse Among Men Who Have Sex with Men: Results from a Respondent Driven Sampling Survey in Nanjing, China, 2008. AIDS Behav. 2013;17:1415-22.

45. Li YZ, Xu JJ, Qian HZ, You BX, Zhang J, Zhang JM, et al. High prevalence of HIV infection and unprotected anal intercourse among older men who have sex with men in China: a systematic review and meta-analysis. BMC Infect Dis. 2014;14:531.

46. State Council AIDS Working Committee Office (SCAWCO). 2012 China AIDS Response Progress Report. Beijing, China: Ministry of Health of the People's Republic of China; 2012. p. 1-70. http://unaids.org.cn/pics/20120614140133. pdf. Accessed on 27 January, 2015. [Context Link].

47. Shang $H, X U$ J, Han X, Spero Li J, Arledge KC, Zhang L. HIV prevention: Bring safe sex to China. Nature. 2012;485:576-7.

48. Emlet CA, Brennan DJ, Brennenstuhl S, Rueda S, Hart TA, Rourke SB. The impact of HIV-related stigma on older and younger adults living with HIV disease: does age matter? AIDS Care. 2014;14:1-9.

\section{Submit your next manuscript to BioMed Central and we will help you at every step:}

- We accept pre-submission inquiries

- Our selector tool helps you to find the most relevant journal

- We provide round the clock customer support

- Convenient online submission

- Thorough peer review

- Inclusion in PubMed and all major indexing services

- Maximum visibility for your research

Submit your manuscript at www.biomedcentral.com/submit 